Article

\title{
Examining the Influence of Cultural Immersion on Willingness to Try Fruits and Vegetables among Children in Guam: The Traditions Pilot Study
}

\author{
Tanisha F. Aflague ${ }^{1, *}$, Rachael T. Leon Guerrero ${ }^{1}$, Treena Delormier ${ }^{2}$, Rachel Novotny ${ }^{3}$, \\ Lynne R. Wilkens ${ }^{4}$ and Carol J. Boushey ${ }^{4}$ (D) \\ 1 College of Natural and Applied Sciences, University of Guam, Mangilao, GU 96923, USA; \\ rachaeltlg@triton.uog.edu \\ 2 School of Human Nutrition, McGill University, Sainte-Anne-de-Bellevue, QC H9X 3L9, Canada; \\ treena.delormier@mcgill.ca \\ 3 Human Nutrition, Food, and Animal Sciences, College of Tropical Agriculture and Human Resources, \\ University of Hawaii at Manoa, Honolulu, HI 96822, USA; novotny@hawaii.edu \\ 4 University of Hawaii Cancer Center, University of Hawaii at Manoa, Honolulu, HI 96813, USA; \\ lynne@cc.hawaii.edu (L.R.W.); cjboushey@cc.hawaii.edu (C.J.B.) \\ * Correspondence: taflague@triton.uog.edu; Tel.: +1-671-735-2026
}

Received: 11 September 2019; Accepted: 29 October 2019; Published: 20 December 2019

\begin{abstract}
This pilot study examined the influence of cultural immersion on willingness to try fruits and vegetables (FV) among children 3-12 years old in three summer camps in Guam with different cultural exposure levels: cultural immersion camp (CIC), high exposure; university day camp (UDC), moderate exposure; and recreational sports camp (RSC), zero exposure. Children, ages 3-12 years old for CIC and UDC and 5-12 years old for RSC, participated: CIC $(n=47)$, UDC $(n=23)$, and RSC ( $n=33)$. Children's willingness to try FV was assessed with the Adapted WillTry tool before and after each program. Whole FV intakes were assessed concurrently using the mobile food record in CIC and UDC. Using multivariate regression, WillTry post-assessment outcomes were modeled adjusting for pre-assessment, child characteristics, exposure, and parent cultural affiliation. Unique to the Adapted WillTry tool are three FV scales, local novel, local common, and imported, which are classified by source (local or imported) and/or familiarity (novel or common). WillTry adjusted mean FV post-scores by highest exposure camp to lowest were 2.2, 2.3, and 2.2 for local novel and 2.6, 2.6, and 2.6 for local common. No differences among camps were significant; however, there was an increase in the willingness to try scores for all FV score types and camps. The Traditions pilot study demonstrated: (1) feasibility of a multi-arm parallel design using existing community programs in limited-resource environments and (2) further examination of nutrition education components and contexts are needed to understand diet behaviors of indigenous populations.
\end{abstract}

Keywords: child intervention; fruit and vegetable intake; Guam

\section{Introduction}

For the higher obesity prevalence rates in indigenous populations, including in Guam, the Chamorros compared to non-Hispanic whites correlate with chronic disease patterns [1]. Sociocultural factors, including traditional dietary habits and the cultural significance of staple foods, have the potential to influence positive health behavioral change [1,2]. Among children, common intervention strategies have been nutrition lessons, fruit and vegetable (FV) exposure or tasting, and peer modeling in school or camp settings [3,4]. Culturally adapted programs have increased children's fruit and 
vegetable $(\mathrm{FV})$ intake and/or preference [3,5]. One study in Guam found a culturally modified nutrition curriculum originally designed for children in the U.S. mainland was feasible and warranted [6].

Children's preferences for FV was shown to be an adequate indicator of FV consumption [7]. A study among children, 3-11 years old (y), in Guam [8] adapted the previously validated WillTry tool that measures children's willingness to try FV [9]. Unique to the Adapted WillTry tool [8] are three FV scales, local novel, local common, and imported, which are classified by source (local or imported) and/or familiarity (novel or common). Children were found to prefer imported FV more than local FV [8]. This finding parallels the nutrition transition [10,11], where diets shifted away from local traditional food systems [12], and highlights a need to examine interventions to improve preference for and intake of local (traditional) FV as a way to increase intake and variety of FV overall.

Culture provides the context of meaning for individual behaviors and is, therefore, a critical element for influential behavioral interventions, such as FV intake, mediated by FV preference $[7,13]$. For many indigenous cultures, the traditional food system and cultural practices around food create opportunities for FV exposure and intake $[6,12,14]$. The traditional food system is food within a culture from local, natural resources [15]. Traditional diets and practices, as well as a sense of ethnic identity or pride among indigenous peoples, have been shown to protect health [15-17].

The Traditions pilot study described here examined the influence of Chamorro cultural immersion on children's willingness to try local FV in Guam. The primary hypothesis was that children attending a cultural immersion camp with culturally adapted nutrition lessons would have a higher Adapted WillTry post-score than children in programs without cultural immersion. A secondary hypothesis was that these same children would also have a higher whole FV intake. This is the first study to examine whether cultural immersion influences children's FV preference and intake in the Pacific.

\section{Materials and Methods}

A pre-post, quasi-experimental design was used to measure outcomes (i.e., willingness to try FV and FV intake) between three existing summer camp programs in Guam: cultural immersion camp (CIC), university day camp (UDC), and recreational sports camp (RSC). This pilot, multi-arm parallel design study cost-effectively used existing programs run by organizations willing to incorporate activities to answer the research question [18].

Children registered in the 2014 study summer camps were eligible to participate. The age range for participants was 3-12 y for CIC and UDC and 5-12 y for RSC. In RSC, only children who were registered for two, 2-week sessions were eligible. These programs each had a registration fee and were open to children of any race and ethnicity. Recruitment materials were included with camp registration packets. The Human Studies Program of the University of Hawaii (as part of the first author's thesis effort) and the University of Guam Committee on Human Subjects approved this study.

The primary intervention was the cultural immersion within CIC. Unique to the Traditions pilot study were culturally adapted nutrition lessons incorporated into CIC and UDC. CIC had a high cultural dose as the Traditions lessons were implemented within the cultural immersion context of the camp. The UDC had moderate cultural dose from the Traditions lessons only. RSC was without both cultural immersion and Traditions lessons, or had zero cultural doses.

The CIC and UDC programs were nearly matched for daily activities (e.g., physical activity, cooking demonstrations/taste testing, crafts) shown in Table 1. The same four Traditions lessons (Table 2) were incorporated into CIC and UDC and delivered by the same educators. These lessons featured local FV and promoted positive associations with eating FV. In CIC, the context of Chamorro cultural traditions, practices, and values was tied to FV. Chamorro is the language, culture, and ethnicity of Guam and the Marianas. In UDC, consuming healthy foods was introduced within a context of nutrition and human physiology. The four lessons were adapted with permission from a culturally relevant Hawaiian nutrition curriculum [19] to make them relevant to Guam and the Chamorro culture. This curriculum complemented Hawaii and Guam Department of Education K-12 Content and Performance Standards. 
Table 1. Summary of the Traditions pilot study camps programs philosophy, subject areas, activities, and language spoken.

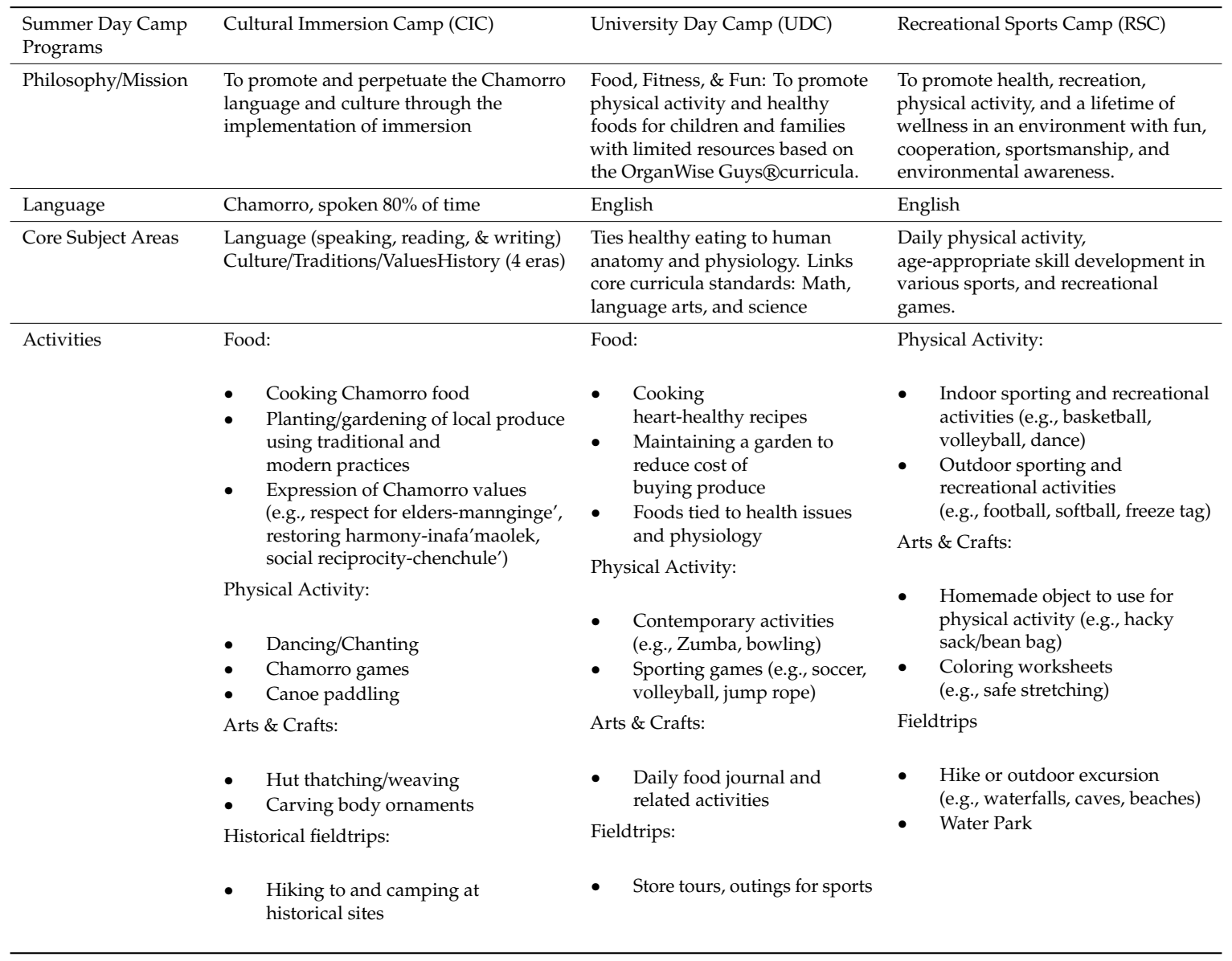

Table 2. Details of the four culturally adapted nutrition lessons, Traditions lessons unique to this study, included local foods incorporated into the cultural immersion camp (CIC) and the university day camp (UDC) programs delivered to children aged 3-12 years old in Guam for the Traditions pilot study.

\begin{tabular}{|c|c|c|c|}
\hline Lesson 1 & Lesson 2 & Lesson 3 & Lesson 4 \\
\hline Food Safety \& Hand Washing & A Foundation for Good Health & Label Detectives & Food Choices for Your Environment \\
\hline Key Concepts: & Key Concepts & & Key Concepts \\
\hline Activities: & Activities: & Activities: & Activities: \\
\hline $\begin{array}{l}\text { 1) Handwashing song, "Biba } \\
\text { Komplianos" (20 s) } \\
\text { 2) Clean (Na'gasgas), Separate } \\
\text { (Na'asangi), Cook (Fatinas), } \\
\text { Chill (Na'manengheng) } \\
\text { Worksheet \& Activity }\end{array}$ & $\begin{array}{l}\text { 1) Close to the source } \\
\text { activity with mångga } \\
\text { (mango) } \\
\text { 2) Exploring the Pacific } \\
\text { Food Guide } \\
\text { 3) Planning a } \\
\text { healthy breakfast }\end{array}$ & $\begin{array}{l}\text { 1) Identifying Red Flag } \\
\text { ingredients of common } \\
\text { snack foods for children } \\
\text { at camp } \\
\text { 2) } \\
\text { Measuring sugar in } \\
\text { popular beverages } \\
\text { in Guam }\end{array}$ & $\begin{array}{l}\text { 1) Comparing food miles between } \\
\text { local banana (chotda/aga' håya) } \\
\text { and banana from US Mainland } \\
\text { (aga' lagu) } \\
\text { 2) Evaluate ways to keep the } \\
\text { environment safe }\end{array}$ \\
\hline $\begin{array}{l}\text { Recipe: } \\
\text { Breadfruit kabobs: steamed } \\
\text { breadfruit dipped in warm } \\
\text { coconut milk }\end{array}$ & $\begin{array}{l}\text { Recipe: } \\
\text { Eggplant (egg) scramble }\end{array}$ & $\begin{array}{l}\text { Recipe: } \\
\text { Papaya and/or mango } \\
\text { smoothie }\end{array}$ & $\begin{array}{l}\text { Recipe: } \\
\text { Soursop popsicles and frozen or } \\
\text { dehydrated star fruit }\end{array}$ \\
\hline
\end{tabular}


The CIC activities perpetuated the Chamorro culture and language through song/chanting, dance, prayer, arts and crafts, cooking, outdoor activities, and gardening based on traditional and contemporary practices. Chamorro was spoken about $80 \%$ of the time, including the delivery of key messages in the Traditions lessons. All activities operated on indigenous values of respect (respetu), love (guaiya), humility (mamåhlao), reciprocity (chenchule'), and restoring harmony (inafa'maolek). The UDC activities focused on promoting healthful present-day recreational activities and global foods. The activities were an extension of the Expanded Food and Nutrition Education Program mission [20]. RSC was exclusively physical activity. Refer to Table 2. Upon completion of the last assessments, educators provided at least one Traditions lesson for registrants at RSC.

Data collection occurred at two assessment periods: before ( \pm 2 weeks) and after ( \pm 1 week) each program. The first assessment was completed at each camp setting. The second was also completed at camp or at a pre-arranged location, such as the children's homes or a child-friendly public space (e.g., the mall).

Adapted WillTry Scores: The Adapted WillTry tool measures children's willingness to try FV and was previously validated for children 3-11 years old in Guam [8,9]. The Adapted WillTry has 3 distinct scales of FV: local novel, local common, and imported. The scores for each scale ranged from 1 to 3 , i.e., least to most willing to try. The Adapted WillTry administration and scoring methods have been described previously [8].

Whole FV Intake: Minimally processed FV (e.g., FV mixed dishes, fruits, vegetables) intake was assessed using the mobile food record ( $m F R$ ) running on iOS7.1.2 on an Apple iPod touch (Apple Inc., Cupertino, CA, USA). The $m F R$ is an app that has been shown to be a useful method for dietary assessment with adolescents [21,22]. This method was also tested and found to be usable for children 3-11 y in Guam [23]. Only participants in CIC and UDC were asked to use the $m F R$ due to a limited number of iPods. Children used the $m F R$ for two consecutive days to capture before and after images of all eating occasions. Instructions on how to use the $m F R$ included taking a practice image of plastic food replicas with a fiducial marker (FM). The FM functioned as a color reference and volume marker of the food. Children were loaned the $m F R$ and 2-4 FMs. Children handled the iPod, FMs, and a charging cord with a waterproof mobile carrying case. When the children returned the $m F R$, researchers asked children to assist with identifying food items that were indistinguishable, e.g., opaque containers, occluded foods; and to recall foods at eating occasions not captured as an image.

Other Measures: Parents completed a questionnaire that included information about the child's age, sex, language spoken, religion, and birthplace. Parent's cultural affiliation was determined using their responses reported on a cultural affiliation questionnaire, which assesses one of four modes of acculturation: traditional, integrated, assimilated, or marginalized [24]. The same scoring system was used as described by Kaholokula and others [24]. Anthropometric assessments were completed at a time designated as least disruptive to camp activities. Height and weight were measured using a portable Seca scale and stadiometer (PE-AIM 101, Perspective Enterprises, Portage, MI, USA) using centimeters and kilograms, respectively. These measurements were converted to body mass index $(\mathrm{BMI})$ as $\left[\mathrm{kg} /(\text { height, } \mathrm{m})^{2}\right]$. Dose of intervention was assessed by recording attendance at camp and at each Traditions lesson.

For participation, all children were given a gift card, in $\$ 5$ or $\$ 10$ denominations, one before and one after camp assessments. Remuneration varied due to the different types and lengths of involvement for participants depending on the camp program.

With a sample size of $n=47$ for CIC and $n=21$ for UDC, with a power of $80 \%$ and a type I error rate of 0.05 (two-sided), the minimum detectable difference in means (MDD) are 0.49 for the local novel score and 0.43 for the local common score [25]. For Model 3, based on the UDC and RSC sample sizes, MDD was 0.52 and 0.49 for local novel and local common, respectively. For comparison of the secondary outcome, post-FV intake, between CIC and UDC, the MDD was 0.63, equivalent to a difference between groups of 1.3 servings per day for an SD of 1.5 . 
Data were entered using a Microsoft Access (Microsoft Corporation, Redmond, WA, USA) tool specifically designed for this study. Double-data entry procedures were used and PROC COMPARE in SAS 9.3 (SAS Institute, Inc., Cary, NC, USA) was performed until both data entries achieved 100\% matching. A trained analyst examined images, identified all whole FV (e.g., FV mixed dishes, fruits, vegetables), and amounts consumed. FV (100\%) juices were excluded [26]. FV intake was calculated by dividing the total FV by the total number of days for which eating occasions were captured using the $m F R$.

Categorical variables were examined using frequencies and percentages and, for continuous variables, means and standard deviations were used. Doses were calculated based on Traditions lessons and camp attendance for CIC and UDC as the sum of days participants attended lessons and camp days divided by the total possible lesson and camp days, respectively. A dichotomous variable was created for high and low doses using the 50th percentile cut point for lessons and camp dose.

The primary outcome was the Adapted WillTry FV post-scores for local novel, local common, and imported FV. The secondary outcome was post-whole FV intake assessed in CIC and UDC only. To examine the primary hypothesis, multiple linear regression models of the Adapted WillTry post-scores (dependent variables) were fit to examine whether and how much the Adapted WillTry scores differed by camp program accounting for potential confounders. A separate model was fit for each type of score: local novel, local common, and imported. Potential confounders were age (i.e., 3-6 y, 7-8 y, 9-12 y), ethnicity (i.e., Chamorro, Chamorro Mixed, Other), sex, parent's cultural affiliation (traditional and integrated; marginalized, $n=2$, or assimilated, $n=1$, were eliminated due to small numbers), BMI (continuous), and attendance (i.e., high and low doses at lessons and camp). The final model excluded potential confounders that had no effect on the results and included Adapted WillTry pre-scores, sex, age, and ethnicity. Covariate-adjusted Adapted WillTry FV post-scores for each camp were computed from the models and differences between camps were evaluated with a global F-test and pairwise tests adjusted for multiple comparisons by the Tukey method. All pairwise comparisons were performed. It was hypothesized that higher cultural dose would lead to higher Adapted WillTry FV post-scores. In addition, $p$-values $<0.05$ were considered statistically significant.

For whole FV intake, a similar multiple linear regression model of the post-FV intake/day was used to examine differences between UDC and CIC. The final FV model was adjusted for pre-FV intake/day, age, ethnicity, and parent's cultural affiliation. In addition, to compare with previously observed incremental trends of willingness to try FV scores [8], unadjusted difference in the pre- and post-assessment scores for each FV scale in each camp are computed from a mixed model of scores regressed on camp program, time (pre and post), and the interaction and accounting for the repeated measures. As recommended by Hochberg and Tamhane [27], all pairwise comparisons were performed. Statistical analyses were conducted using IBM SPSS Statistics version 21 (IBM Corporation, Armonk, NY, USA).

\section{Results}

A total of 104 children met the eligibility criteria and agreed to participate. There were 47, 23, and 34 children from CIC, UDC, and RSC, respectively, and percent loss to follow up from the respective camps were $2 \%, 9 \%$, and $3 \%$ (refer to Figure 1). Children participating in both assessment periods included 29 boys (4-11 years old) and 70 girls (3-12 years old) or 96\% (99/104) of the original enrollees. The majority of the children were Chamorro as reported by parents. Refer to Table 3. For the secondary outcome of FV intake, analysis was limited to 14 boys and 43 girls that completed at least two-days of food recording using the $m F R$. 


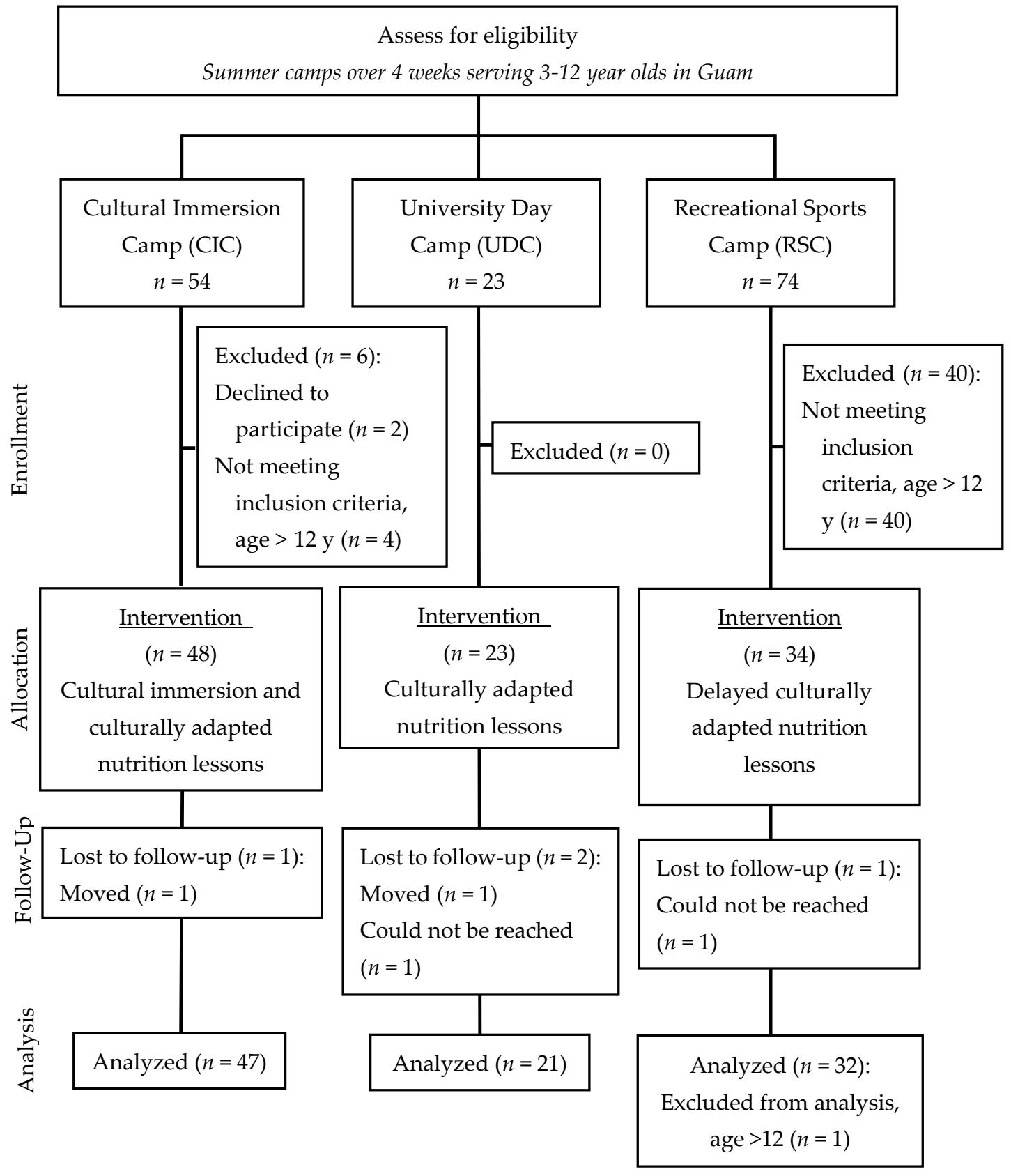

Figure 1. Assessment for eligibility, enrollment, allocation, follow-up, and analysis of children 3-12 years of age participating in the Traditions pilot study. 
Table 3. Characteristics of children in the Traditions pilot study in Guam by camp program, ages 3-12 years old that completed the Adapted WillTry at pre and post $(n=99)$.

\begin{tabular}{|c|c|c|c|c|}
\hline Child Characteristics & $n$ & $\operatorname{CIC}^{1}(n=46)$ & $\operatorname{UDC}^{2}(n=21)$ & $\operatorname{RSC}^{3}(n=32)$ \\
\hline Sex & & & $n$ (percent, \%) & \\
\hline Boys & 29 & $17(37)$ & $1(5)$ & $11(34)$ \\
\hline Girls & 70 & $29(63)$ & $20(95)$ & $21(66)$ \\
\hline \multicolumn{5}{|l|}{ Ethnic Group } \\
\hline Chamorro, only & 53 & $25(54)$ & $12(57)$ & $16(50)$ \\
\hline Chamorro, mixed & 33 & $21(46)$ & $3(14)$ & $9(28)$ \\
\hline Other & 12 & $0(0)$ & $6(29)$ & $6(19)$ \\
\hline No response & 1 & $0(0)$ & $0(0)$ & $1(3)$ \\
\hline \multicolumn{5}{|l|}{ Age Group, years } \\
\hline $3-6$ & 28 & $17(37)$ & $1(5)$ & $10(31)$ \\
\hline $7-8$ & 39 & $14(30)$ & $8(38)$ & $17(53)$ \\
\hline $9-12$ & 32 & $15(33)$ & $12(57)$ & $5(16)$ \\
\hline \multicolumn{5}{|l|}{ Weight status, $\mathrm{BMI}^{+}$percentile } \\
\hline Underweight, $<5$ th percentile & 3 & $2(4)$ & $1(5)$ & $0(0)$ \\
\hline Healthy weight, 5 to $<85$ th percentile & 66 & $28(61)$ & $16(76)$ & $22(69)$ \\
\hline Overweight, $\geq 85$ to $<95$ th percentile & 16 & $9(20)$ & $3(14)$ & $4(12)$ \\
\hline Obese, $\geq 95$ th percentile & 14 & $7(15)$ & $1(5)$ & $6(19)$ \\
\hline \multicolumn{5}{|l|}{ Parent's cultural affiliation } \\
\hline Traditional & 15 & $6(13)$ & $5(23)$ & $4(12)$ \\
\hline Integrated & 79 & $38(83)$ & $14(67)$ & $27(84)$ \\
\hline Marginalized & 2 & $2(4)$ & $0(0)$ & $0(0)$ \\
\hline Assimilated & 1 & $0(0)$ & $1(5)$ & $0(0)$ \\
\hline No response & 2 & $0(0)$ & $1(5)$ & $1(3)$ \\
\hline Pre-Assessments & & Mean \pm SD & Mean \pm SD & Mean \pm SD \\
\hline \multicolumn{5}{|l|}{ Adapted WillTry (unadjusted) scores } \\
\hline Local Novel & & $2.2 \pm 0.6$ & $2.4 \pm 0.4$ & $2.2 \pm 0.6$ \\
\hline Local Common & & $2.4 \pm 0.6$ & $2.5 \pm 0.4$ & $2.6 \pm 0.4$ \\
\hline Imported & & $2.6 \pm 0.4$ & $2.9 \pm 0.2$ & $2.8 \pm 0.3$ \\
\hline Whole FV $\ddagger$ (servings) & & $0.6 \pm 0.5^{\mathrm{a}}$ & $1.2 \pm 1.1^{\mathrm{b}}$ & $\mathrm{n} / \mathrm{a}^{\#}$ \\
\hline
\end{tabular}

${ }^{1}$ CIC, Cultural Immersion Camp (high dose); ${ }^{2}$ UDC, University Day Camp (moderate dose); ${ }^{3}$ RSC, Recreational Sports Camp (zero dose). ${ }^{\mathrm{a}} n=47 ;{ }^{\mathrm{b}} n=23 .{ }^{\dagger}$ Body Mass Index; ${ }^{\ddagger}$ fruits and vegetables; ${ }^{\#}$ not applicable.

There were no significant differences at $p<0.05$ between camp programs for the Adapted WillTry local novel and local common FV post-scores (refer to Table 4). The lack of significant differences among these scores remained after adjustment in multivariate analyses. The arm with the lowest cultural dose had similar post-means as the other group and even the highest score for the imported category (Table 4).

Table 4. Adjusted post means for Adapted WillTry score and fruit and vegetable intake among children, 3-12 years old, in Guam by Traditions pilot study camp program.

\begin{tabular}{cccccccc}
\hline & \multicolumn{4}{c}{ Post Means $^{\mathbf{1}}$} & \multicolumn{3}{c}{$p$-Value for Pairwise Comparisons ${ }^{3}$} \\
\hline & $\begin{array}{c}\text { CIC } \\
(\boldsymbol{n}=\mathbf{4 7})\end{array}$ & $\begin{array}{c}\text { UDC } \\
(\boldsymbol{n}=\mathbf{2 1})\end{array}$ & $\begin{array}{c}\text { RSC } \\
(\boldsymbol{n}=\mathbf{3 2})\end{array}$ & $\begin{array}{c}\text { Global } \\
\boldsymbol{p} \text {-value }\end{array}$ & $\begin{array}{c}\text { CIC vs. } \\
\text { UDC }\end{array}$ & $\begin{array}{c}\text { CIC vs. } \\
\text { RSC }\end{array}$ & $\begin{array}{c}\text { USC vs. } \\
\text { RSC }\end{array}$ \\
\hline $\begin{array}{c}\text { Local Novel Adapted } \\
\text { WillTry score }\end{array}$ & 2.2 & 2.3 & 2.2 & 0.87 & 0.89 & 1.00 & 0.87 \\
$\begin{array}{c}\text { Local Common Adapted } \\
\quad \text { WillTry Score }\end{array}$ & 2.6 & 2.6 & 2.6 & 0.97 & 1.00 & 0.96 & 0.99 \\
$\begin{array}{c}\text { Imported Adapted } \\
\text { WillTry Score }\end{array}$ & 2.7 & 2.6 & 2.8 & 0.06 & 0.56 & 0.27 & 0.06 \\
Whole FV (servings) & $0.3^{\mathrm{a}}$ & $0.7^{\mathrm{b}}$ & $\mathrm{n} / \mathrm{a} \neq$ & 0.25 & 0.25 & $\mathrm{n} / \mathrm{a}$ & $\mathrm{n} / \mathrm{a}$ \\
\hline
\end{tabular}

${ }^{1}$ Adjusted by regression for age, sex, ethnicity, and pre-assessment scores. ${ }^{2}$ Based on F-test comparing all three camps, except for Whole FV (i.e., CIC and UDC) ${ }^{3}$ Adjusted for multiple comparisons by the Tukey method. ${ }^{\text {a }} n=$ $38 ;{ }^{\mathrm{b}} n=19 .{ }^{\ddagger}$ not applicable. 
For whole FV intake, the unadjusted means for whole FV intake/day at pre- and post-assessments were $1.2(n=23)$ and $0.7(n=19)$ for UDC and $0.6(n=47)$ and $0.3(n=38)$ for CIC, respectively. There were no significant differences in post-whole FV intakes between the camps after adjusting for the potential confounders in the final regression model $(p=0.86)$. The null relationship holds when only considering the 57 individuals with pre and post $\mathrm{FV}$ intakes.

For all FV score type and camps, there was an increase in the willingness to try FV scores. The exception was for UDC for Imported FV. Specifically, for Local Novel, the mean changes are 0.06 for CIC, 0.03 for UDC, and 0.04 for RSC. The corresponding changes for Local Common are $0.16,0.11$, and 0.05 , and for Imported are $0.05,-0.04$, and 0.06 .

\section{Discussion}

This report represents the first quasi-experiment to examine whether cultural immersion can positively influence children's willingness to try local FV. With regard to increasing willingness to try local novel and local common FV for any of the camp comparisons, there were no statistically significant changes in either direction, i.e., decrease or increase. Interestingly, RSC had a similar or greater change in willingness to try imported FV than the other camps. Although there were no significant differences across camps, overall the Traditions lessons moved the FV scores higher regardless of camp, which is similar to what was observed in past studies using other methodologies.

The change in means by camp demonstrated the consistency and robustness of the Adapted WillTry scores with regard to maintaining a trend (from high to low) in willingness to try imported, local common, and local novel FV that was observed in a previous study [8]. This indicates that indeed children are more willing to try imported FV than local FV and furthermore are more willing to try local FV familiar to them than other local FV that are unfamiliar to them. The Traditions pilot study represents the first time to use the Adapted WillTry tool in an intervention to capture changes in food preferences. Moreover, the lower preference for local FV underscores that interventions aimed to improve willingness to try local FV may hold promise for improving overall FV intake as children already have a high preference for imported FV.

The Traditions pilot study involved community-based summer camp programs that have been in operation for, at least, seven years in Guam. This pilot study design highlights services and activities available in Guam and extends a community-based approach by enhancing local programs within their expectation and capacity. Community-based approaches are suitable for indigenous populations involving processes drawing on local knowledge to develop interventions and have been successful in the South Pacific [28]. The risk, however, is that camp programs themselves were not intended to incorporate willingness to try FV. However, the fidelity of lessons were ensured by having the same educators deliver the Traditions lessons.

In general, we did not have complete control of the activities within each camp that contributed to limitations of this pilot study. All camps had predetermined schedules (e.g., duration). UDC and RSC schedules overlapped restricting FV diet assessment to only UDC due to the limited number of iPods. All camp programs had 2- or 4-week programs limiting the intervention period to 4-weeks. Using the existing community-based programs, study participants and their families had self/family-selected for the three summer camp programs. Similarly, the small sample size was due to only some of the registered camp children participating in the research activities.

Within the CIC, the use of the Chamorro language to deliver the Traditions lessons may have been a limitation since CIC staff assisted with implementation and translation of lessons and nutrition concepts. Language could be a potential barrier as children likely are not fluent and are learning the Chamorro language while at camp. Nonetheless, the context of the camp in which the lessons were delivered encompassed Chamorro values and practices that may have contributed to the shift in local common FV preference. Perhaps the activities and values that were used to promote and perpetuate the Chamorro culture in the CIC do not directly involve FV, but generally all the traditional 
and contemporary foods of the culture. Future research involving cultural immersion should include methods and instruments that can help to capture the cultural context and impact of cultural exposure.

The traditional Chamorro food system may be transforming in turn shifting food choices parallel to the nutrition transition, creating challenges to promoting traditional local food, like FV, today. Therefore, altering children's food preferences may extend beyond a cultural immersion program limited to the activities within the program and limited by time. However, these programs preserve traditional knowledge underpinning the value of local FV and revitalize customs that have the potential to improve local FV preference and ultimately intake. As demonstrated in this pilot study: (1) a multi-parallel design is feasible in a limited-resource environment with existing community programs, especially when examining cultural immersion that is place-based and (2) nutrition education, like Traditions lessons, increased willingness to try FV in all camps. Therefore, further examination of nutrition education components and educational contexts are needed to better understand willingness to try foods and diet behaviors of indigenous populations.

\section{Conclusions}

The Traditions pilot study demonstrated: (1) feasibility of a multi-arm parallel design using existing community programs in limited-resource environments and (2) further examination of nutrition education components and contexts are needed to understand diet behaviors of indigenous populations.

Author Contributions: Conceptualization, T.F.A. and C.J.B.; methodology, T.F.A. and C.J.B.; software, C.J.B.; validation, C.J.B. and L.R.W.; formal analysis, T.F.A., C.J.B., and L.R.W.; investigation, T.F.A.; resources, C.J.B. and R.N.; data curation, T.F.A.; writing-original draft preparation, T.F.A. and C.J.B.; writing-review and editing, R.T.L.G., R.N., and T.D.; visualization, T.F.A., C.J.B., R.T.L.G., and T.D.; supervision, C.J.B. and R.T.L.G.; project administration, T.F.A.; funding acquisition, R.N. and C.J.B. All authors have read and agreed to the published version of the manuscript.

Funding: This research was funded by Agriculture and Food Research Initiative Grant No. 2011-68001-30335 from the USDA National Institute of Food and Agricultural Science Enhancement Coordinated Agricultural Program.

Acknowledgments: The authors thank the summer camps and the families and children who participated in the study; Kokua Hawaii Foundation for permission to adapt 'AINA In Schools nutrition curriculum lessons; Rosae Calvo, for her contributions to the study; and Ed Delp and doctoral students from the VIPER Lab at Purdue University at the College of Electrical and Computer Engineering for facilitating access and use of the mobile food record.

Conflicts of Interest: The authors declare no conflict of interest. The funders had no role in the design of the study; in the collection, analyses, or interpretation of data; in the writing of the manuscript, or in the decision to publish the results.

\section{References}

1. Institute of Medicine. Preventing Childhood Obesity: Health in the Balance; The National Academy Press: Washington, DC, USA, 2005.

2. Huang, T.T.; Drewnowski, A.; Kumanyika, S.K.; Glass, T.A. A systems-oriented multi-level framework for addressing obesity in the 21st century. Prev. Chronic Dis. 2009, 6, 1-10.

3. Govula, C.; Kattelman, K.; Ren, C. Culturally appropriate nutrition lessons increased fruit and vegetable consumption in American Indian children. Top. Clin. Nutr. 2007, 22, 239-245. [CrossRef]

4. Blanchette, L.; Brug, J. Determinants of fruit and vegetable consumption among 6-12 year-old children and effective interventions to increase consumption. J. Hum. Nutr. Dietet. 2005, 18, 431-443. [CrossRef] [PubMed]

5. Baranowski, T.; Baranowski, J.C.; Cullen, K.W.; Thompson, D.I.; Nicklas, T.; Zakeri, I.E.; Rochson, J. The fun, food, and fitness project (FFFP): The Baylor GEMS pilot study. Ethn. Dis. 2003, 13, S30-S39.

6. Pobocik, R.S.; Montgomery, D. Roff Gemlo, L. Modification of a school-based nutrition education curriculum to be culturally relevant for western pacific islanders. J. Nutr. Educ. 1998, 30, 164-169. [CrossRef]

7. Ventura, A.K.; Worobey, J. Early influences on the development of food preferences. Curr. Biol. 2013, 23, 401-408. [CrossRef] 
8. Aflague, T.F.; Leon Guerrero, R.T.; Boushey, C.J. Adaptation and evaluation of the WillTry tool to assess willingness to try fruits and vegetables among children 3-11y in Guam. Prev. Chronic Dis. 2014, 11. [CrossRef]

9. Thomson, J.L.; McCabe-Sellers, B.J.; Strickland, E.; Lovera, D.; Nuss, H.J.; Yadrick, K.; Duke, S.; Bogle, M.L. Development and evaluation of WillTry. An instrument for measuring children's willingness to try fruits and vegetables. Appetite 2010, 54, 465-472. [CrossRef]

10. Snowdon, W.; Raj, A.; Reeve, E.; Guerrero, R.L.T.; Fesaitu, J.; Catine, K.; Guignet, C. Processed Foods Available in the Pacific Islands; Global Health: Edinburgh, UK, 2013.

11. Popkin, B.M. Contemporary nutritional transition: Determinants of diet and its impact on body composition. Proc. Nutr. Soc. 2011, 70, 82-91. [CrossRef]

12. Kuhnlein, H.V.; Receveur, O. Dietary change and traditional food systems of indigenous peoples. Annu. Rev. Nutr. 1996, 16, 417-442. [CrossRef]

13. Satia-About a, J.; Patterson, E.R.; Neuhouser, M.L.; Elder, J. Dietary acculturation applications to nutrition research and dietetics. JADA 2002, 102, 1105-1118.

14. Torsch, V. Living the health transition among the Chamorros of Guam. Pac. Health Dialog 2002, 9, $263-274$. [PubMed]

15. Kuhnlein, H.; Erasmus, B.; Creed-Kanashiro, H.; Englberger, L.; Okeke, C.; Turner, N.; Allen, L.; Bhattacharjee, L. Indigenous peoples' food systems for health: Finding interventions that work. Public Health Nutr. 2007, 9, 1013-1019. [CrossRef]

16. Renzaho, A.M.N.; Swinburn, B.; Burns, C. Maintenance of traditional cultural orientation is associated with lower rates of obesity and sedentary behaviours among African migrant children to Australia. Int. J. Obes. 2008, 32, 594-600. [CrossRef] [PubMed]

17. Lind, C.; Mirchandani, G.G.; Castrucci, B.C.; Chavez, N.; Handler, A.; Hoelscher, D.M. The effects of acculturation on healthy lifestyle characteristics among Hispanic fourth-grade children in Texas public schools, 2004-2005. J. Sch. Health 2012, 82, 166-174. [CrossRef]

18. Harris, A.D.; McGregor, J.C.; Perencevich, E.N.; Furuno, J.P.; Zhu, J.; Peterson, D.E.; Finkelstein, J. The use and interpretation of quasi-experimental studies in medical informatics. J. Am. Med. Inform. Assoc. 2006, 13, 16-23. [CrossRef]

19. Kokua Hawai'i Foundation Resources: AINA Nutrition Education Unit Overview. Kokua Hawaii Foundation: Haleiwa, HI, USA. Available online: http://kokuahawaiifoundation.org/aina/resources (accessed on 8 March 2014).

20. Auld, G.; Baker, S.; Conway, L.; Dollahite, J.; Lambea, M.C.; McGirr, K. Outcome effectiveness of the widely adopted EFNEP curriculum Eating Smart Being Active. J. Nutr. Educ. Behav. 2015, 47, 19-27. [CrossRef]

21. Daugherty, B.L.; Schap, T.E.; Ettienne-Gittens, R.; Zhu, F.M.; Bosch, M.; Delp, E.J.; Ebert, D.S.; Kerr, D.A.; Boushey, C.J. Novel technologies for assessing dietary intake: Evaluating the usability of a mobile telephone food record among adults and adolescents. J. Med. Internet Res. 2012, 14, e58. [CrossRef]

22. Six, B.L.; Schap, T.E.; Zhu, F.M.; Mariappan, A.; Bosch, M.; Delp, E.J.; Ebert, D.S.; Kerr, D.A.; Boushey, C.J. Evidence-based development of a mobile telephone food record. J. Am. Diet. Assoc. 2010, 110, 74-79. [CrossRef]

23. Aflague, T.F.; Boushey, C.J.; Leon-Guerrero, R.T.; Ahmad, Z.; Kerr, D.A.; Delp, E.J. Feasibility and use of the mobile food record for capturing eating occasions among children ages 3-10 years in Guam. Nutrients. 2015, 7, 4403-4415. [CrossRef]

24. Kaholokula, J.K.; Iwane, M.K.; Nacapoy, A.H. Effects of received racism and acculturation on hypertension in Native Hawaiians. Hawaii Med. J. 2010, 69, 11. [PubMed]

25. Friedman, L.M.; Furberg, C.D.; DeMets, D.L. Fundamentals of Clinical Trials; John Wright, PSG Inc.: Boston, MA, USA, 1980.

26. Chiuve, S.E.; Fung, T.T.; Rimm, E.B.; Hu, F.B.; McCullough, M.L.; Wang, M.; Stampfer, M.J.; Willett, W.C. Alternative dietary indices both strongly predict risk of chronic disease. J. Nutr. 2012, 142, 1009-1018. [CrossRef] [PubMed] 
27. Hochberg, Y.; Tamhane, A.C. Multiple Comparison Procedures; John Wiley \& Sons: New York, NY, USA, 1987.

28. Fialkowski, M.K.; DeBaryshe, B.; Bersamin, A.; Nigg, C.; Leon Guerrero, R.; Rojas, G.; Areta, A.A.R.; Vargo, A.; Belyeu-Camacho, T.; Castro, R.; et al. A community engagement process identifies environmental priorities to prevent early childhood obesity: The children's healthy living (CHL) program for remote underserved populations in the US affiliated Pacific Islands, Hawaii and Alaska. Matern. Child Health J. 2013, 18, 2261-2274. [CrossRef] [PubMed]

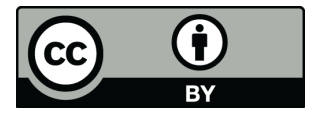

(C) 2019 by the authors. Licensee MDPI, Basel, Switzerland. This article is an open access article distributed under the terms and conditions of the Creative Commons Attribution (CC BY) license (http://creativecommons.org/licenses/by/4.0/). 